University of Nebraska - Lincoln

DigitalCommons@University of Nebraska - Lincoln

French Language and Literature Papers

Modern Languages and Literatures, Department

2021

\title{
Éloge de la fuite
}

Stéphane Bikialo

Follow this and additional works at: https://digitalcommons.unl.edu/modlangfrench

Part of the French and Francophone Language and Literature Commons

This Article is brought to you for free and open access by the Modern Languages and Literatures, Department of at DigitalCommons@University of Nebraska - Lincoln. It has been accepted for inclusion in French Language and Literature Papers by an authorized administrator of DigitalCommons@University of Nebraska - Lincoln. 


\section{Stéphane Bikialo}

\section{Éloge de la fuite}

[Ce texte a paru dans Lydie Salvayre, éd. Stéphane Bikialo, Classiques Garnier, 2020.]

«Si on y allait? »

(fin de $B W$ )

Je pars.

Toujours il dit Je pars, je me tire.

Il aime le mouvement de partir. Il se fout de l'endroit à atteindre, ce qu'il aime c'est partir, c'est déclarer qu'il part. Il dit qu'il va écrire, un jour, l'éloge de la fuite. $(B W)$

C'est BW qui le dit, et c'est Lydie Salvayre qui l'écrit, inaugurant avec cet ouvrage, paru en 2009, une série qu'elle décrira ainsi dans Hymne (2011) : « le temps presse et [...] il me faut aller désormais vers ce qui, entre tout, m'émeut et m'affermit, vers tout ce qui m'augmente ». Ce sera les 7 femmes en 2013, et sa mère et Bernanos en 2014 avec Pas pleurer, roman dont j'emprunterai le dispositif énonciatif

Published in Lydie Salvayre, maintenant même, ed. Warren Motte (Lincoln, NE : Zea Books, 2021). doi: 10.32873/unl.dc.zea.1290 
d'ouverture («C'est Bernanos qui le dit ») afin de suivre cet « éloge de la fuite » qui s'écrit dans l'œuvre de Lydie Salvayre, d'un roman à l'autre, d'une fuite à l'autre. Et cet éloge de la fuite, j'ai eu envie de l'écrire avec les personnages de ses livres, avec BW en particulier car tout le livre est une variation sur le fait de partir, mais aussi avec Montaigne, « toujours botté et prêt à partir ». Souvent, chez Lydie Salvayre, il faut non seulement être prêt à partir, mais savoir partir, « car il faut des forces immenses pour s'élancer, contrairement à ce que postulent les assis qui assimilent la fuite à je ne sais quelle veulerie de l'âme » $(B W)$. Si en effet on peut partir pour le geste ou le mouvement même de partir, on peut aussi partir pour fuir, partir pour refuser, partir pour « disparaître de soi »...

Tout commence par « ce que pudiquement, on appela la Retirada » (Pas pleurer), qui fût à la fois une fuite et un refus : la fuite devant les violences commises par les franquistes, le refus de se soumettre aux fascistes triomphants. C'est ce que firent les parents de Lydie Salvayre quand l'Espagne devint franquiste. Cette fuite originelle est racontée dans Pas pleurer, mais aussi dans La Puissance des mouches et en arrière-plan de plusieurs autres romans (La Méthode Mila notamment). La fuite est aussi une solution pour signifier son refus de la société de la consommation et du spectacle contemporaine, comme y insiste Rémy Oudghiri dans Petit Éloge de la fuite hors du monde (Arléa, 2014) en prenant appui notamment sur la vie et l'œuvre de Pascal Quignard : «Au moment où le spectacle et la mise en scène de soi s'imposent comme des compétences centrales dans nos sociétés, le retrait du monde représente peut-être la dernière option pour ceux qui veulent protéger leur intimité ». C'est ce que fait la narratrice de Contre, qui décide de fuir après dix 
ans passés dans « une étrange république » où « les alcools sont fameux, ainsi que les fromages et l'esprit bien connu de collaboration $»$ :

Ma vie, je la portais comme on porte son sac.

Je sus qu'il fallait fuir.

Je pris les autoroutes. Je traversai des mers, des fleuves et quatre continents. J'avançais vite et sans me retourner. Comme on s'évade après un meurtre. Parfois, je fus tentée de renoncer à ce voyage et revenir à mon sommeil abject. Mais jamais, cependant, je ne relâchais mon rythme. Je voulais m'écarter d'un pays où les hommes s'éteignent à force de se soumettre.

Quand la vie devient survie, quand les semblables ne sont plus des frères, il faut savoir partir donc. Mieux vaut donc fuir, chercher un homme ou autre chose, que de se soumettre, que de rester dans un « sommeil abject », comme le fera la narratrice de Portrait de l'écrivain en animal domestique en se laissant anesthésier par le luxe prodigué par Tobold : « Je vivais dans une douceur soporifique que j'aurais réprouvée sévèrement six mois auparavant au nom des convictions politiques dont alors je me drapais ». Mieux vaut fuir que se laisser dominer par la «puissance des mouches » qui « empêchent notre âme d'agir » et « mangent notre corps » ( $L a$ Puissance des mouches). C'est le choix que font le narrateurpersonnage de Tout Homme est une nuit, Anas, et Mîna, car il leur est impossible d'habiter dans ce village où leur présence puis leur amour cristallise la haine : « habiter près d'une frontière, c'est plus commode pour s'enfuir » (dernière phrase du roman). Augustin, le fils de Marcelin le tenancier du café des sports, réussit lui aussi à partir (pas très loin certes) à la fois pour fuir et pour vivre. 
Partir, c'est ce que ne sont pas capables de faire les habitués du café des sports, sauf verbalement et ivres :

Émile, à demi assommé, se prit alors à hurler $\mathrm{Ah}$ ! putain ! si j'avais trente ans de moins, j'enverrais tout dinguer ! Tout ! Le pavillon qui m'a bouffé les économies de toute ma vie et que je n'ai pas fini de payer ! Tout, ma femme, les mioches, le garage, tout ce caca! hurla-t-il. Et j'irais faire la fiesta à Las Vegas ! [...] Moi aussi j'aimerais foutre le camp de chez moi, avoua Dédé, lui aussi bien éméché mais qui contrôlait encore.

Sans cette capacité à partir, à chercher autre chose, c'est le refus de l'autre qui émerge, comme Marcelin, Dédé et les autres, dont Montaigne aurait pu dire : «j'ai honte de voir nos hommes enivrés de cette sotte humeur de s'effaroucher des formes contraires aux leurs : il leur semble être hors de leur élément quand ils sont hors de leur village. Où qu'ils aillent, ils se tiennent à leurs façons, et abominent les étrangères » (Essais). Ce rejet de l'autre était déjà présent, en arrière-plan, dans La Méthode Mila, où les Roms, et Mila qui les soutient, cristallisent la haine des habitués du café des Ormes, ce qui aboutit au viol de Perline, la fille de Mila.

Lydie Salvayre montre à quel point les immobiles, ceux qui ne fuient pas car ils sont en position de pouvoir, sont bornés dans leur espace comme dans leur esprit. Ces personnages hiératiques, ce sont souvent des pères : le père de Josep et Montse puis Diego dans Pas pleurer tous les deux « DESPOTIQUES », le père du narrateur de La Puissance des mouches, etc. Ce sont aussi les puissants, ceux qui ont le pouvoir de l'argent : les dirigeants de La Médaille, Tobold dans Portrait de l'écrivain en animal domestique, l'huissier dans 
La Compagnie des spectres, qui ne voit pas plus loin que le bout de la loi. « Nous devons, quoiqu'il nous en coûte, nous cantonner à la Loi, disparaître derrière elle, être son ombre, rien de plus », recommande l'huissier en chef dans Quelques Conseils utiles aux élèves huissiers. Ceux-là ont les idées fixes et les mots morts comme dans La Médaille (« L'ouvrier vrai est modeste »; « Vivez votre travail dans sa dimension spirituelle ! »). Ceux-là font partir, expulsent comme ce même huissier, Maître Échinard, dans La Compagnie des spectres. Face à ces ennemis, il faut partir. Ou alors il faut les faire partir. Le narrateur de La Puissance des mouches fera le choix de tuer son père plutôt que de fuir, et Rose Mélie et Louisiane s'associeront pour chasser Maître Échinard à la fin du roman :

Avant d'ouvrir la porte, maman, doctement, déclara, citant Marcus Caton, Il faut faire avec le méchant comme avec l'ouragan le marin. Et sur ces belles paroles, nous le jetâmes dehors. Dans l'ouragan.

C'est la force ironique de Quelques Conseils utiles aux élèves huissiers que de donner des techniques (ne pas allumer la lumière, faire un concert de casseroles, invectiver son chat, bombarder de photographies au flash ...) pour faire partir ces réincarnations contemporaines des miliciens vichystes. L'huissier devient la figure emblématique de celui qui reste, qui poursuit son « inventaire du désastre » (premier titre envisagé pour La Compagnie des spectres) d'une maison à l'autre mais aussi d'une époque à l'autre, avec la rigidité de tous les réalistes, statiques, tièdes comme Descartes et sa méthode dénoncée dans La Méthode Mila.

Ces personnages aux corps et aux idées arrêtées sont souvent, quand ils ne sont pas dans la police (Passage à 
l'ennemie), les habitués des cafés qui déversent leur haine de l'autre, des étrangers, des nomades : ceux du café des sports (Tout Homme est une nuit), ceux du café des Ormes (La Méthode Mila), ceux du café des Platanes (La Puissance des mouches, La Compagnie des spectres). À la manière de Marie Cosnay dans Comment on expulse: Responsabilités en miettes, Lydie Salvayre restitue, d'un livre à l'autre, une chaîne de responsabilités de ce rejet de ceux qui sont partis et qui sont rejetés pour cela.

La fuite, le départ peut avoir plus nettement comme cause le refus. C'est Montaigne qui le dit : « l'autre cause qui me convie à ces promenades, c'est la disconvenance aux mœurs présentes de notre État » (Essais, livre III, chap. IX). C'est Josep cette fois qui le dit, et qui le fera avec Montse, sa sœur : « Je vais foutre le camp, dit-il, quitter ce trou à rat » (Pas pleurer). Quitter cette maison avec un père « facha » et ce village qui se laissera convaincre par le réalisme de Diego, c'est refuser la tiédeur révolutionnaire, la servitude volontaire des exploités et le cynisme des exploitants. C'est BW qui le dit et le fait aussi, son départ de la maison d'édition qu'il a créée étant aussi lié à son « horreur des ladres », à son refus de rester dans ce trou à rat que devient l'édition : «BW vomit la tiédeur et, par-dessus tout, la tiédeur littéraire », « car BW, qui est âgé de 62 ans a connu l'édition du temps où elle était, dit-il, une culture, une résistance ». Dans un entretien, Roland Barthes affirme que la seule contestation qu'aucun pouvoir ne tolère jamais, c'est « la contestation par le retrait. On peut affronter un pouvoir par attaque ou par défense ; mais le retrait, c'est ce qu'il y a de moins assimilable par une société » (« La crise du désir », repris dans Le Grain de la voix). Il faut donc savoir partir comme manière de dire NON, comme la narratrice de Contre, mais 
aussi comme l'Inspecteur Arjona, qui demande sa radiation, à la fin de Passage à l'ennemie, pour « partir » avec sa fiancée « quelque part, n'importe où, loin des familles et de leurs meurtres » pour se consacrer à Dulcinée mais aussi à « l'art d'écrire ». Celles-là ou ceux-là ne renoncent pas : en disant NON, ils disent aussi OUI, ils croient que ça mérite de partir, comme dirait Camus selon lequel l'homme révolté est « un homme qui dit non. Mais s'il refuse, il ne renonce pas : c'est aussi un homme qui dit oui, dès son premier mouvement » (L'Homme révolté).

La fuite se fait brusquement (« je me tire », dit BW ; «J'avançais vite et sans me retourner », lit-on dans Contre), elle est impulsive, elle est un élan, un saut hors de la rangée des assassins aurait dit Kafka. Le départ n'est pas le voyage, tout au moins pas un de ces voyages à la durée et aux espaces (escales, but) prédéterminés ; ainsi les personnages des Belles Âmes ne partent pas, ils voyagent, dans « les pourrissoirs de l'Europe » à la recherche moins du réel que de la réalité qu'ils ont observée à travers les médias. Ce sont des touristes, pas des « parteurs »-j'aurais bien employé « marcheurs » en référence aux travaux sur la marche de David Le Breton, mais il faudra d'abord désintoxiquer ce mot annexé par les macronistes en 2017-, auxquels il manque cette « humeur avide des choses nouvelles et inconnues » (Montaigne, Essais), comme Odile Coiffard qui se dit « fort déçue par ce qu'elle découvre. Ni voitures incendiées. Ni cabines téléphoniques saccagées. Ni tags sur les murs. Rien. $\mathrm{Qu}$ 'une trentaine de blocs ternes de part et d'autre d'une autoroute ».

Il est une forme de fuite qui ne se fait pas dans la rapidité (même si le départ peut être brusque) mais qui privilégie la lenteur : il s'agit de ce que l'anthropologue David Le 
Breton nomme « disparaître de soi » dans un ouvrage paru en 2015. Disparaître de soi, c'est faire l'expérience de la « blancheur », « cet état d'absence à soi plus ou moins prononcé, le fait de prendre congé de soi sous une forme ou sous une autre à cause de la difficulté ou de la pénibilité d'être soi » (Disparaître de soi). Par cette fuite ou disparition, il s'agit de refuser l'injonction très contemporaine à « être soi-même », injonction qui fige dans une identité comme Pascal Quignard le dénonce selon Rémy Oudghiri. Chez Lydie Salvayre, l'identité est toujours en doute (« je, qui ça? » trouve-t-on dans l'épigraphe empruntée à Beckett de Portrait de l'écrivain en animal domestique), car elle ne peint pas l'être mais peint le passage, « le vif du vivant » comme elle l'identifie chez Picasso. C'est pourquoi ses ouvrages abondent en formes de fuites hors du monde qui sont aussi des moyens de disparaître de soi : la lecture (de Pascal ou de Descartes par exemple), la folie (ou ce qui apparaît comme telle pour les autres) dans La Conférence de Cintegabelle, La Compagnie des spectres, La Puissance des mouches...

Tobold, suite à la découverte d'une inscription injurieuse à son égard, envisage de tout laisser tomber, de « disparaître de soi » :

Tobold était las de ce monde de merde, il me le confia d'une voix exténuée, il voulait vivre loin des banques de merde, loin des banquiers de merde et de leurs épouses de merde éprises d'art premier et de ce que, dans leur langue, elles appelaient littérature, il voulait vivre près des vaches de son enfance, crues et vivantes.

Mais Tobold n'atteindra pas la blancheur, il se reconvertira dans le charity business, ce qui lui permet tout à la fois de se construire une image de soi valorisante, de faire taire 
ses ennemis et de continuer son travail de domination par l'argent.

Disparaître de soi devient nécessaire dans une société où l'on est en permanence obligé d'être à la hauteur, où l'exigence d'être soi-même, dans sa cohérence, l'emporte sur le fait de l'être, dans sa diversité. BW rejoint les analyses de David Le Breton lorsqu'il « philosophile » :

Car vivre c'est quitter, pas d'autre issue pour l'homme, je cite la hagada, s'enflamme BW, passionné qu'il est des textes religieux. Car vivre c'est quitter père et mère et tout ce qui nous lie jusqu'à nous étrangler. Vivre c'est se quitter, c'est savoir être soi et échapper à soi, c'est savoir être soi et un autre que soi. (BW)

Le narrateur de La Puissance des mouches renonce à fuir mais parcourt des dizaines de kilomètres « le long des chemins de campagne » :

Je sors et prends le chemin qui mène à la forêt de chênes rouvres. Je marche longtemps. Je cherche un endroit sec. Je me couche sous un chêne. Je suis longtemps des yeux la course des nuages. Je ne pense à rien. Je me sens bien.

La marche est centrale dans de nombreux romans de Lydie Salvayre, notamment pour ces personnages qui ont besoin de « partir », ponctuellement ou définitivement. David Le Breton, dans un entretien au Temps (12 juillet 2016) au sujet de ses ouvrages sur la marche-Éloge de la marche en 2000, actualisé en 2012 sous le titre Marcher (éloge des chemins et de la lenteur) — souligne que « la marche est peutêtre la manière la plus démocratique pour retrouver des 
moments de paix, d'harmonie, de disparition de soi. Mais une disparition de soi mesurée. On part quelques heures ou quelques jours, et les portables ont souvent du mal à fonctionner dans les endroits isolés. L'immense succès sociologique de la marche tient à cette suspension des contraintes de l'identité. Sur les sentiers, plus personne ne sait qui vous êtes, vous n'avez de compte à rendre à personne. Vous marchez à votre rythme, vous vous arrêtez ... Personne ne vous rappelle à l'ordre pour un rendement que vous devez accomplir ». C'est ce que fait le narrateur de La Déclaration, qui « marche longtemps »: «j'espère fatiguer ma tristesse, m'oublier dans les jambes, me perdre dans le vert ». C'est ce que fait aussi le narrateur-personnage de Tout Homme est une nuit, quittant son lieu d'habitation et sa femme Lucile pour s'installer dans un village non nommé près de $\mathrm{Ba}-$ rogne-ne cherchez pas sur une carte mais sous le mot, pour y entendre la rogne et la bassesse-en raison de sa maladie :

$\mathrm{Au}$ lieu donc de m'amender, au lieu de me grandir, au lieu de me rendre aimable aux autres avant de définitivement leur faire mes adieux, la maladie distillait un poison dans mon cœur qui me faisait l'humeur amère et me rendait insupportable à Lucile comme à tous mes proches.

Pour toutes ces raisons-là, j'avais décidé de partir.

Partir, pour lui, c'est prendre rendez-vous avec soimême, c'est découvrir la forêt même à trente ans, « gagner en liberté » contre l'air vicié des méchants, mais aussi contre la sollicitude des proches. C'est aussi savoir se séparer de soi. Savoir partir c'est aussi savoir profiter de ce qui compte lorsqu'on a une maladie grave, et s'il faut finir, que ce soit en beauté : 
Je me disais que si la maladie l'emportait, je souhaitais qu'elle le fît en beauté, dans un lieu de beauté, dans un lieu d'éblouissante lumière, dans un lieu de profuses couleurs, dans le bleu le vert le rose et le lilas.

Je voulais bien finir avant d'appareiller vers l'ultime océan.

C'est le narrateur de Tout Homme est une nuit qui parle. Lui qui fera l'épreuve du fait que, comme l'écrit Éric Hazan dans Faire mouvement, « il est difficile de créer un îlot d'harmonie dans un océan de domination », lui qui réussit, comme de nombreux personnages de Lydie Salvayre, et comme elle-même depuis quelques années, réussit à se trouver une « arrière-boutique ». C'est Montaigne qui le dit : « il se faut réserver une arrière-boutique toute nôtre, toute franche, en laquelle nous établissons notre vraie liberté et principale retraite et solitude » (Essais, I, XXIX). 